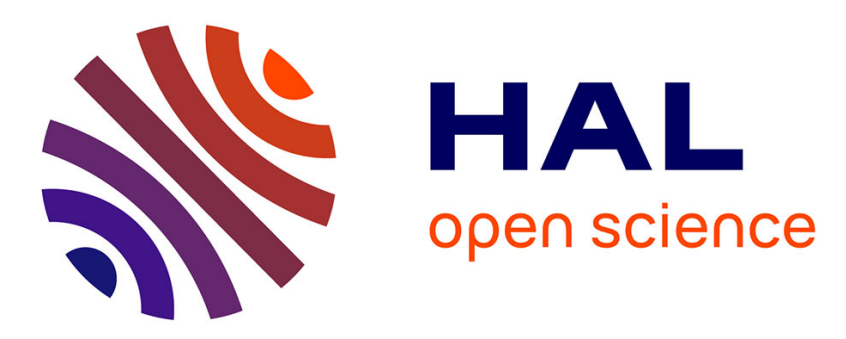

\title{
Painting-to-3D Model Alignment Via Discriminative Visual Elements
}

\author{
Mathieu Aubry, Bryan C. Russell, Josef Sivic
}

\section{To cite this version:}

Mathieu Aubry, Bryan C. Russell, Josef Sivic. Painting-to-3D Model Alignment Via Discriminative Visual Elements. ACM Transactions on Graphics, 2014, 33 (2), pp.14. 10.1145/2591009 . hal$00863615 \mathrm{v} 3$

\section{HAL Id: hal-00863615 https://inria.hal.science/hal-00863615v3}

Submitted on 21 Aug 2014

HAL is a multi-disciplinary open access archive for the deposit and dissemination of scientific research documents, whether they are published or not. The documents may come from teaching and research institutions in France or abroad, or from public or private research centers.
L'archive ouverte pluridisciplinaire HAL, est destinée au dépôt et à la diffusion de documents scientifiques de niveau recherche, publiés ou non, émanant des établissements d'enseignement et de recherche français ou étrangers, des laboratoires publics ou privés. 


\title{
Painting-to-3D Model Alignment via Discriminative Visual Elements
}

\author{
MATHIEU AUBRY \\ INRIA and TU München \\ BRYAN C. RUSSELL \\ Intel Labs \\ and \\ JOSEF SIVIC \\ INRIA
}

\begin{abstract}
This article describes a technique that can reliably align arbitrary 2D depictions of an architectural site, including drawings, paintings, and historical photographs, with a 3D model of the site. This is a tremendously difficult task, as the appearance and scene structure in the 2D depictions can be very different from the appearance and geometry of the 3D model, for example, due to the specific rendering style, drawing error, age, lighting, or change of seasons. In addition, we face a hard search problem: the number of possible alignments of the painting to a large 3D model, such as a partial reconstruction of a city, is huge. To address these issues, we develop a new compact representation of complex 3D scenes. The 3D model of the scene is represented by a small set of discriminative visual elements that are automatically learned from rendered views. Similar to object detection, the set of visual elements, as well as the weights of individual features for each element, are learned in a discriminative fashion. We show that the learned visual elements are reliably matched in 2D depictions of the scene despite large variations in rendering style (e.g., watercolor, sketch, historical photograph) and structural changes (e.g., missing scene parts, large occluders) of the scene. We demonstrate an application of the proposed approach to automatic rephotography to find an approximate viewpoint of historical paintings and photographs with respect to a 3D model of the site. The proposed alignment procedure is validated via a human user study on a new database of paintings and sketches spanning several sites. The results demonstrate that our algorithm produces significantly better alignments than several baseline methods.
\end{abstract}

Categories and Subject Descriptors: I.3.10 [Artificial Intelligence]: Vision and Scene Understanding-3D/stereo scene analysis; I.4.10 [Image Processing and Computer Vision]: Image Representation—Statistical

This work was partly supported by the ANR project SEMAPOLIS, EIT ICT Labs, the MSR-INRIA Laboratory, and Google.

Authors' addresses: M. Aubry (corresponding author), INRIA WILLOW Project Team, Departement d'Informatique de l'Ecole Normale Superieure, ENS/INRIA/CNRS UMR 8548, France and TU München; email: mathieu.aubry@m4x.org; B. C. Russell, Intel Labs; J. Sivic, INRIA WILLOW Project Team, Departement d'Informatique de l'Ecole Normale Superieure, ENS/INRIA/CNRS UMR 8548, France; email: josef.sivic@ens.fr.

Permission to make digital or hard copies of all or part of this work for personal or classroom use is granted without fee provided that copies are not made or distributed for profit or commercial advantage and that copies bear this notice and the full citation on the first page. Copyrights for components of this work owned by others than ACM must be honored. Abstracting with credit is permitted. To copy otherwise, or republish, to post on servers or to redistribute to lists, requires prior specific permission and/or a fee. Request permissions from permissions@acm.org.

(c) 2014 ACM 0730-0301/2014/03-ART14 $\$ 15.00$

DOI: http://dx.doi.org/10.1145/2591009
General Terms: Algorithms, Design, Experimentation

Additional Key Words and Phrases: 3D alignment, rephotography, paintings, sketches, historical photographs, 3D models, CAD models

ACM Reference Format:

Mathieu Aubry, Bryan C. Russell, and Josef Sivic. 2014. Painting-to-3D model alignment via discriminative visual elements. ACM Trans. Graph. 33, 2. Article 14 (March 2014) 14 pages.

DOI: http://dx.doi.org/10.1145/2591009

\section{INTRODUCTION}

In this work we seek to automatically align historical photographs and nonphotographic renderings, such as paintings and line drawings, to a 3D model of an architectural site. Specifically, we wish to establish a set of point correspondences between local structures on the $3 \mathrm{D}$ model and their respective $2 \mathrm{D}$ depictions. The established correspondences will in turn allow us to find an approximate viewpoint of the 2D depiction with respect to the 3D model. We focus on depictions that are, at least approximately, perspective renderings of the 3D scene. We consider complex textured 3D models obtained by recent multiview stereo reconstruction systems [Furukawa et al. 2010] as well as simplified models obtained from 3D modeling tools such as Trimble 3D Warehouse. Example results are shown in Figure 1.

Why is this task important? First, nonphotographic depictions are plentiful and comprise a large portion of our visual record. We wish to reason about them, and aligning such depictions to reference imagery (via a 3D model in this case) is an important step towards this goal. Second, such technology would open up a number of exciting computer graphics applications that currently require expensive manual alignment of $3 \mathrm{D}$ models to various forms of 2D imagery. Examples include interactive visualization of a 3D site across time and different rendering styles [Debevec et al. 1996; Levin and Debevec 1999], model-based image enhancement [Kopf et al. 2008], annotation transfer for augmented reality [Snavely et al. 2006], inverse procedural 3D modeling [Aliaga et al. 2007; Musialski et al. 2012], or computational rephotography [Rapp 2008; Bae et al. 2010]. Finally, reliable automatic image-to-3D model matching is important in domains where reference 3D models are often available, but may contain errors or unexpected changes (e.g., something built/destroyed) [Bosché 2010], such as urban planning, civil engineering, or archaeology.

The task of aligning 3D models to 2D nonphotographic depictions is extremely challenging. As discussed in prior work [Russell 


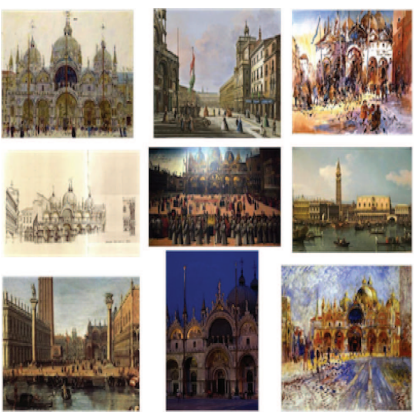

(a) Internet paintings and drawings

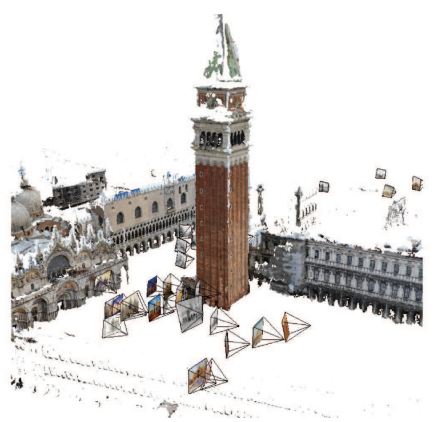

(b) painting viewpoints
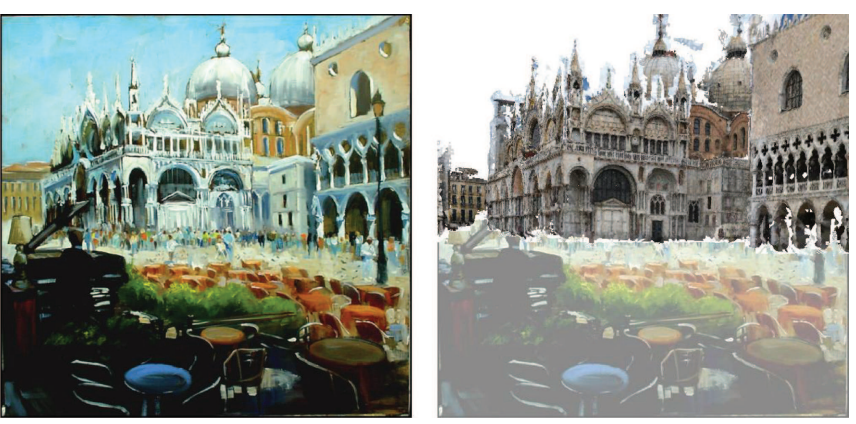

(c) aligned painting to 3D model

Fig. 1. Our system automatically aligns and recovers the viewpoint of paintings, drawings, and historical photographs to a 3D model of an architectural site. Painting in (c) courtesy of Podi Lawrence.

et al. 2011; Shrivastava et al. 2011], local feature matching based on interest points (e.g., SIFT [Lowe 2004]) often fails to find correspondences across paintings and photographs. First, the rendering styles across the two domains can vary considerably. The scene appearance (colors, lighting, texture) and geometry depicted by the artist can be very different from the rendering of the $3 \mathrm{D}$ model, for example, due to the depiction style, drawing error, or changes in the geometry of the scene. Second, we face a hard search problem. The number of possible alignments of the painting to a large 3D model, such as a partial reconstruction of a city, is huge. Which parts of the painting should be aligned to which parts of the 3D model? How does one search over the possible alignments?

To address these issues we introduce the idea of automatically discovering discriminative visual elements for a $3 \mathrm{D}$ scene. We define a discriminative visual element to be a mid-level patch that is rendered with respect to a given viewpoint from a 3D model with the following properties: (i) it is visually discriminative with respect to the rest of the "visual world" represented here by a generic set of randomly sampled patches, (ii) it is distinctive with respect to other patches in nearby views, and (iii) it can be reliably matched across nearby viewpoints. We employ modern representations and recent methods for discriminative learning of visual appearance which have been successfully used in recent object recognition systems. Our method can be viewed as "multiview geometry [Hartley and Zisserman 2004] meets part-based object recognition [Felzenszwalb et al. 2010]"- - here we wish to automatically discover the distinctive object parts for a large 3D site.

We discover discriminative visual elements by first sampling candidate mid-level patches across different rendered views of the $3 \mathrm{D}$ model. We cast the image matching problem as a classification task over appearance features with the candidate mid-level patch as a single positive example and a negative set consisting of a large set of "background" patches. Note that a similar idea has been used in learning per-exemplar distances [Frome et al. 2007] or perexemplar Support Vector Machine (SVM) classifiers [Malisiewicz et al. 2011] for object recognition and cross-domain image retrieval [Shrivastava et al. 2011]. Here we apply per-exemplar learning for matching mid-level structures between images.

For a candidate mid-level patch to be considered a discriminative visual element, we require that: (i) it has a low training error when learning the matching classifier, and (ii) it is reliably detectable in nearby views via cross-validation. Critical to the success of operationalizing the preceding procedure is the ability to efficiently train linear classifiers over Histogram of Oriented Gradients (HOG) features [Dalal and Triggs 2005] for each candidate mid-level patch, which has potentially millions of negative training examples. In contrast to training a separate SVM classifier for each mid-level patch, we change the loss to a square loss, similar to Bach and Harchaoui [2008] and Gharbi et al. [2012], and show that the solution can be computed in closed form, which is computationally more efficient as it does not require expensive iterative training. In turn, we show that efficient training opens up the possibility to evaluate the discriminability of millions of candidate visual elements densely sampled over all the rendered views. We further show how our formulation is related to recent work that performs Linear Discriminant Analysis (LDA) by analyzing a large set of negative training examples and recovering the sample mean and covariance matrix that decorrelates the HOG features [Hariharan et al. 2012; Gharbi et al. 2012].

The output for each discriminative visual element is a trained classifier. At runtime, for an input painting, we run the set of trained classifiers in a sliding-window fashion across different scales. Detections with high responses are considered as putative correspondences with the 3D model, from which camera resectioning is performed. We show that our approach is able to scale to a number of different 3D sites and handles different input rendering styles. Moreover, we are able to handle different types of 3D models, such as 3D CAD models and models constructed using multiview stereo [Furukawa and Ponce 2010]. To evaluate our alignment procedure, we introduce a database of paintings and sketches spanning several sites and perform a user study where human subjects are asked to judge the goodness of the output alignments. We compare with several baseline methods, such as SIFT on rendered views, the coarse viewpoint retrieval step of Russell et al. [2011], and Exemplar SVM [Shrivastava et al. 2011], and show that our algorithm produces more good alignments than the baselines. Moreover, we evaluate our matching step on the benchmark dataset of Hauagge and Snavely [2012] and show improvement over local symmetry features [Hauagge and Snavely 2012] and several alternative matching criteria for our system.

\section{PRIOR WORK}

This section reviews prior work on aligning $3 \mathrm{D}$ models to $2 \mathrm{D}$ imagery.

Alignment using local features. Local invariant features and descriptors such as SIFT [Lowe 2004] represent a powerful tool for matching photographs of the same at least lightly textured scene despite changes in viewpoint, scale, illumination, and partial occlusion. Example applications include 3D reconstruction [Snavely 
et al. 2006], image mosaicing [Szeliski 2006], visual search [Sivic and Zisserman 2003], visual localization [Schindler et al. 2007], and camera tracking [Ballan et al. 2010] to list but a few. Large 3D scenes, such as a portion of a city [Li et al. 2012], can be represented as a 3D point cloud with associated local feature descriptors extracted from the corresponding photographs [Sattler et al. 2011]. Camera pose of a given query photograph can be recovered from 2D-to-3D correspondences obtained by matching the appearance of local features verified using geometric constraints [Hartley and Zisserman 2004]. However, appearance changes beyond the modeled invariance, such as significant perspective distortions, nonrigid deformations, nonlinear illumination changes (e.g., shadows), weathering, change of seasons, structural variations, or a different depiction style (photograph, painting, sketch, drawing) cause local feature-based methods to fail [Hauagge and Snavely 2012; Shrivastava et al. 2011; Russell et al. 2011]. Greater insensitivity to appearance variation can be achieved by matching the geometric or symmetry pattern of local image features [Chum and Matas 2006; Hauagge and Snavely 2012; Shechtman and Irani 2007], rather than the local features themselves. However, such patterns have to be detectable and consistent between the matched views.

Alignment using contours. Contour-based 3D-to-2D alignment methods [Huttenlocher and Ullman 1987; Lowe 1987] rely on detecting edges in the image and aligning them with projected 3D model contours. Such approaches are successful if object contours can be reliably extracted both from the 2D image and the $3 \mathrm{D}$ model. A recent example is the work on photograph localization using semi-automatically extracted skylines matched to clean contours obtained from rendered views of digital elevation models [Baboud et al. 2011; Baatz et al. 2012]. Contour matching was also used for aligning paintings to $3 \mathrm{D}$ meshes reconstructed from photographs [Russell et al. 2011]. However, contours extracted from paintings and real-world 3D meshes obtained from photographs are noisy. As a result, the method requires a good initialization with a close-by viewpoint. In general, reliable contour extraction is a hard and yet unsolved problem.

Alignment by discriminative learning. Modern image representations developed for visual recognition, such as HOG descriptors [Dalal and Triggs 2005], represent 2D views of objects or object parts [Felzenszwalb et al. 2010] by a weighted spatial distribution of image gradient orientations. The weights are learned in a discriminative fashion to emphasize object contours and de-emphasize nonobject, background contours and clutter. Such a representation can capture complex object boundaries in a soft manner, avoiding hard decisions about the presence and connectivity of imaged object edges. Learned weights have also been shown to emphasize visually salient image structures matchable across different image domains, such as sketches and photographs [Shrivastava et al. 2011]. Similar representation has been used to learn architectural elements that summarize a certain geospatial area by analyzing (approximately rectified) 2D street-view photographs from multiple cities [Doersch et al. 2012]. Also related is contemporary work that utilizes similar representation for scene [Juneja et al. 2013] and action [Jain et al. 2013] classification.

Building on discriminatively trained models for object detection, we develop a compact representation of 3D scenes suitable for alignment to 2D depictions. In contrast to Doersch et al. [2012] and Shrivastava et al. [2011] who analyze 2D images, our method takes advantage of the knowledge and control over the 3D model to learn a set of mid-level 3D scene elements robust to a certain amount of

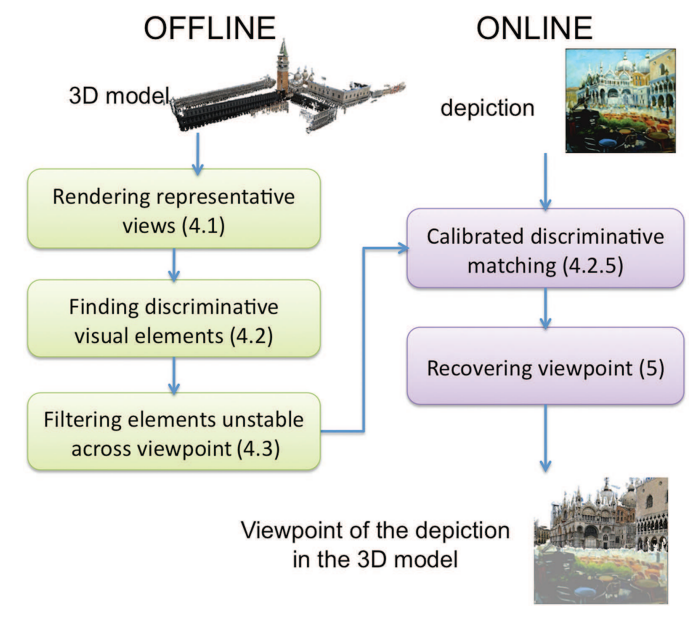

Fig. 2. Approach overview. In the offline stage (left) we summarize a given $3 \mathrm{D}$ model as a collection of discriminative visual elements learned from rendered views of the site. In the online stage (right) we match the learned visual elements to the input painting and use the obtained correspondences to recover the camera viewpoint with respect to the $3 \mathrm{D}$ model.

viewpoint variation and capable of recovery of the (approximate) camera viewpoint. We show that the learned mid-level scene elements are reliably detectable in $2 \mathrm{D}$ depictions of the scene despite large changes in appearance and rendering style.

\section{APPROACH OVERVIEW}

The proposed method has two stages: first, in an offline stage we learn a set of discriminative visual elements representing the architectural site; second, in an online stage a given unseen query painting is aligned with the $3 \mathrm{D}$ model by matching with the learned visual elements. The proposed algorithm is summarized in Figure 2.

In detail, the input to the offline stage is a 3D model of an architectural site. The output is a set of view-dependent visual element detectors able to identify specific structures of the 3D model in various types of 2D imagery. The approach begins by rendering a set of representative views of the $3 \mathrm{D}$ model. Next, a set of visual element detectors is computed from the rendered views by identifying scene parts that are discriminative and can be reliably detected over a range of viewpoints. During the online stage, given an input $2 \mathrm{D}$ depiction, we match with the learned visual element detectors and use the top-scoring detections to recover a coarse camera viewpoint, which can be further refined using dense pixel-wise matching.

\section{FINDING DISCRIMINATIVE VISUAL ELEMENTS FOR 3D-TO-2D MATCHING}

We seek to identify elements of the 3D model that are reliably detectable in arbitrary 2D depictions. To achieve this we build on discriminative learning techniques to identify visually distinctive mid-level scene structures in rendered views of the 3D model. Each rendered view is represented by densely sampled patches at multiple scales, with each patch represented by a Histogram of Oriented Gradient (HOG) descriptor [Dalal and Triggs 2005]. We use the publicly available implementation of HOG from Felzenszwalb et al. [2010]. We only use the contrast-insensitive portion of the HOG descriptor on a $10 \times 10$ grid of cells with 8 orientations within each cell, which results in an 800-dimensional descriptor. The HOG descriptor is 


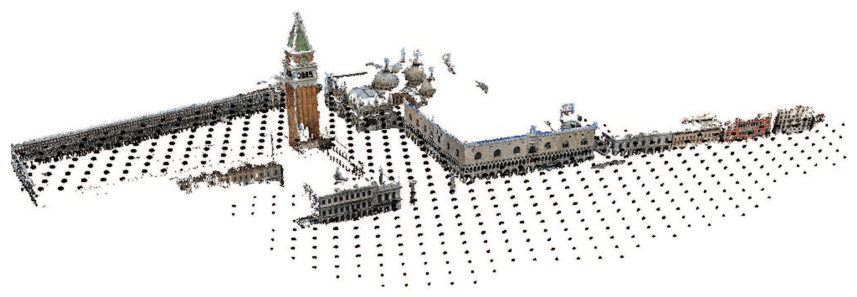

Fig. 3. Example sampled viewpoints. Camera positions are sampled on the ground plane on a regular $100 \times 100$ grid. 24 camera orientations are used for each viewpoint. Cameras not viewing any portion of the 3D model are discarded. This procedure results in about 45,000 valid views for the depicted 3D model.

forgiving to small drawing errors thanks to its spatial and orientation binning. In addition, we use a contrast-insensitive HOG to enhance the capability of matching across different depiction styles. A visual element detector is defined as a linear classifier trained from a single patch as a positive example and a large number of "background" patches as negative examples, similar to an exemplar Support Vector Machine (SVM) [Malisiewicz et al. 2011; Shrivastava et al. 2011]. Every rendered view has thousands of potential visual elements and the task is to identify those that are distinct and hence likely to be detectable in different depictions. For example, a specific tower on the building may be distinctive for the site, whereas a patch in the middle of a gray wall may not. We define a discriminability criterion directly related to the quality of the trained linear classifier and show it can be evaluated efficiently in a per-pixel manner to rank millions of candidate visual elements from all rendered views. Furthermore, to enhance robustness of the trained detectors we discard detectors unstable across changes in viewpoint. In summary, the algorithm proceeds in the following three steps: (i) render a set of representative viewpoints of the 3D site; (ii) efficiently find discriminative candidate elements in all rendered views and learn a detector for each element; (iii) filter element detectors that are unstable across small changes in viewpoint. The three steps are detailed next.

\subsection{Rendering Representative Views}

The aim is to extract from the 3D model a set of view-dependent $2 \mathrm{D}$ descriptors suitable for alignment to $2 \mathrm{D}$ depictions. This is achieved by sampling representative views of the 3D model and learning visual element detectors from the rendered appearance in the sampled views. We sample possible views of the 3D model in a similar manner to Baatz et al. [2012], Irschara et al. [2009], and Russell et al. [2011]. First, we identify the ground plane and corresponding vertical direction. The camera positions are then sampled on the ground plane on a regular grid. For each camera position we sample 12 possible horizontal camera rotations assuming no in-plane rotation of the camera. For each horizontal rotation we sample 2 vertical rotations (pitch angles). Views where less than $5 \%$ of the pixels are occupied by the $3 \mathrm{D}$ model are discarded. This procedure results in 7,000-45,000 views depending on the size of the $3 \mathrm{D}$ site. Example sampled camera positions are shown in Figure 3. Note that the rendered views form only an intermediate representation and can be discarded after visual element detectors are extracted. We render views from the 3D model by adapting the publicly available OpenGL code from Russell et al. [2011] to work with our models. The renderer simply ray casts and samples colors from the textured models against a white background, and does not explicitly reason about illumination effects, such as shadows or

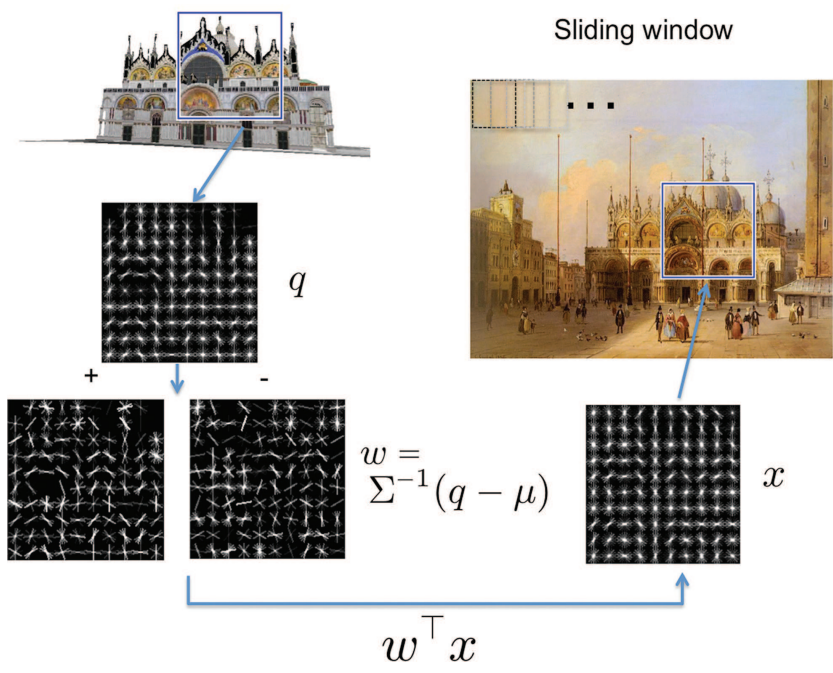

Fig. 4. Matching as classification. Given a region and its HOG descriptor $q$ in a rendered view (top left) the aim is to find the corresponding region in a painting (top right). This is achieved by training a linear HOG-based sliding-window classifier using $q$ as a single positive example and a large number of negative data. The classifier weight vector $w$ is visualized by separately showing the positive $(+)$ and negative $(-)$ weights at different orientations and spatial locations. The best match $x$ in the painting is found as the maximum of the classification score.

specularities (although the textured models may implicitly include this information).

\subsection{Finding Discriminative Visual Elements}

We wish to find a set of mid-level visual elements for the given 3D site that are discriminative. In the following, we formulate image matching as a discriminative classification task and show that, for a specific choice of loss function, the classifier can be computed in closed form without computationally expensive iterative training. In turn, this enables efficient training of candidate visual element detectors corresponding to image patches that are densely sampled in each rendered view. The quality of the trained detector (measured by the training error) is then used to select only the few candidate visual elements that are the most discriminative in each view (have the lowest training error). Finally, we show how the learned visual elements are matched to the input painting, and relate the proposed approach to other recent work on closed-form training of HOGbased linear classifiers [Gharbi et al. 2012; Hariharan et al. 2012].

4.2.1 Matching as Classification. The aim is to match a given rectangular image patch $q$ (represented by a HOG descriptor [Dalal and Triggs 2005]) in a rendered view to its corresponding image patch in the painting, as illustrated in Figure 4. Instead of finding the best match measured by the Euclidean distance between the descriptors, we train a linear classifier with $q$ as a single positive example (with label $y_{q}=+1$ ) and a large number of negative examples $x_{i}$ for $i=1$ to $N$ (with labels $y_{i}=-1$ ). The matching is then performed by finding the patch $x^{*}$ in the painting with the highest classification score

$$
s(x)=w^{\top} x+b,
$$

where $w$ and $b$ are the parameters of the linear classifier. Note that $w$ denotes the normal vector to the decision hyperplane and $b$ is a scalar offset. Compared to the Euclidean distance, the classification 
score (1) measures a form of similarity, that is, a higher classification score indicates higher similarity between $x$ and $q$. In addition, the learned $w$ weights the components of $x$ differently. This is in contrast to the standard Euclidean distance where all components of $x$ have the same weight. Note that a similar idea was used in learning per-exemplar distances [Frome et al. 2007] or per-exemplar SVM classifiers [Malisiewicz et al. 2011] for object recognition and crossdomain image retrieval [Shrivastava et al. 2011]. Here, we build on this work and apply it to image matching using mid-level image structures.

Parameters $w$ and $b$ are obtained by minimizing a cost function of the following form

$$
E(w, b)=L\left(1, w^{T} q+b\right)+\frac{1}{N} \sum_{i=1}^{N} L\left(-1, w^{T} x_{i}+b\right),
$$

where the first term measures the loss $L$ on the positive example $q$ (also called "exemplar") and the second term measures the loss on the negative data. Note that for simplicity we ignore in Eq. (2) the regularization term $\|w\|^{2}$, but the regularizer can be easily added in a similar manner to Bach and Harchaoui [2008] and Gharbi et al. [2012]. We found, however, that adding the regularizer did not result in a significant change in matching performance. A particular case of the exemplar-based classifier is the Exemplar SVM [Malisiewicz et al. 2011; Shrivastava et al. 2011], where the loss $L(y, s(x))$ between the label $y$ and predicted score $s(x)$ is the hinge loss $L(y, s(x))=\max \{0,1-y s(x)\}$ [Bishop 2006]. For Exemplar SVM cost (2) is convex and can be minimized using iterative algorithms [Fan et al. 2008; Shalev-Shwartz et al. 2011].

4.2.2 Selection of Discriminative Visual Elements via Least Squares Regression. So far we have assumed that the position and scale of the visual element $q$ in the rendered view is given. As storing and matching all possible visual elements from all rendered views would be computationally prohibitive, the aim here is to automatically select a subset of the visual elements that are the most discriminative. First, we note that the optimal value of the cost (2) characterizes the separability of a particular candidate visual element $q$ from the (fixed) negative examples $\left\{x_{i}\right\}$ and hence can be used for measuring the degree of discriminability of $q$. However, when using a hinge loss as in Exemplar SVM, optimizing (2) would be expensive to perform for thousands of candidate elements in each rendered view. Instead, similarly to Bach and Harchaoui [2008] and Gharbi et al. [2012], we take advantage of the fact that in the case of square loss $L(y, s(x))=(y-s(x))^{2}$ the $w_{L S}$ and $b_{L S}$ minimizing (2) and the optimal cost $E_{L S}^{*}$ can be obtained in closed form as

$$
\begin{gathered}
w_{L S}=\frac{2}{2+\|\Phi(q)\|^{2}} \Sigma^{-1}(q-\mu), \\
b_{L S}=-\frac{1}{2}(q+\mu)^{T} w_{L S}, \\
E_{L S}^{*}=\frac{4}{2+\|\Phi(q)\|^{2}},
\end{gathered}
$$

where $\mu=\frac{1}{N} \sum_{i=1}^{N} x_{i}$ denotes the mean of the negative examples, $\Sigma=\frac{1}{N} \sum_{i=1}^{N}\left(x_{i}-\mu\right)\left(x_{i}-\mu\right)^{\top}$ their covariance and

$$
\|\Phi(q)\|^{2}=(q-\mu)^{\top} \Sigma^{-1}(q-\mu),
$$

the squared norm of $q$ after the "whitening" transformation

$$
\Phi(q)=\Sigma^{-\frac{1}{2}}(q-\mu) .
$$
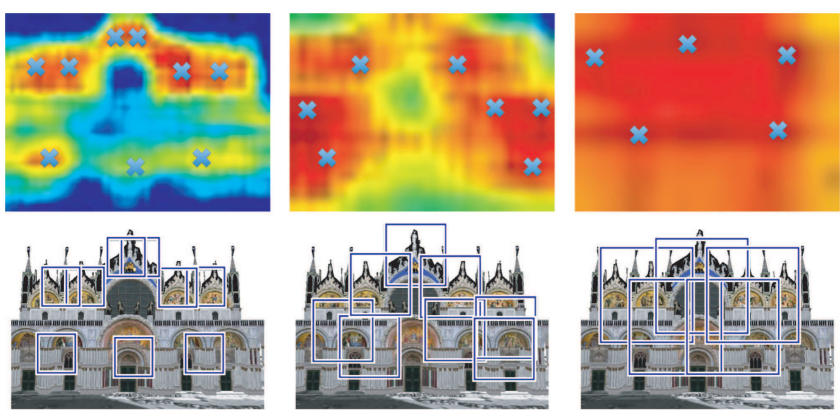

Fig. 5. Selection of discriminative visual elements. First row: discriminability scores shown as a heat map for three different scales. Red indicates high discriminability. Blue indicates low discriminability. The discriminability is inversely proportional to the training cost of a classifier learned from a patch at the particular image location. Second row: example visual elements at the local maxima of the discriminability scores. The corresponding local maxima are also indicated using " $x$ " in the heat maps above.

We can use the value of the optimal cost (5) as a measure of the discriminability of a specific $q$. If the training cost (error) for a specific candidate visual element $q$ is small, the element is discriminative. If the training cost is large the candidate visual element $q$ is not discriminative. This observation can be translated into a simple and efficient algorithm for ranking candidate element detectors based on their discriminability. In practice, we evaluate the squared "whitened" norm $\|\Phi(q)\|^{2}$ of each candidate element $q$, which is inversely proportional to the training cost. If the whitened norm is high the candidate element is discriminative, whereas if the whitened norm is low the candidate element is not discriminative. Given a rendered view, we consider as candidates visual element detectors of all patches that are local maxima (in scale and space) of the norm of their whitened HOG descriptor, $\|\Phi(q)\|^{2}$. Nonmaximum suppression is performed using a threshold of 0.1 on the standard ratio of area intersection over union between two neighboring patches. After this nonmaximum suppression, all remaining patches across all views are ranked according to the same whitened norm criterion. Illustration of multiscale discriminative visual element selection for an example rendered view is shown in Figure 5.

4.2.3 Relation to Linear Discriminant Analysis (LDA). Recent works [Gharbi et al. 2012; Hariharan et al. 2012] have shown that linear HOG-based object detectors computed analytically using Linear Discriminant Analysis (LDA) can reach similar object detection accuracy as detectors learned by expensive iterative SVM training. The distribution of positive and negative data points is assumed to be Gaussian, with mean vectors $\mu_{p}$ and $\mu_{n}$, respectively. The covariance matrix $\Sigma_{p}=\Sigma_{n}=\Sigma$ is assumed to be the same for both positive and negative data. Under these Gaussian assumptions, the decision hyperplane can be obtained via a ratio test in closed form. Applying this approach to our image matching setup, we estimate $\mu_{n}$ and $\Sigma$ from a large set of HOG descriptors extracted from patches that are sampled from a set of ("negative") photographs independent from all sites considered in this work. $\mu_{p}$ is set to be a specific single HOG descriptor $q$ of the particular positive example patch in the given rendered view. Parameters $w_{L D A}$ and $b_{L D A}$ of the linear classifier defining the matching score (1)

$$
s_{L D A}(x)=w_{L D A}^{T} x+b_{L D A},
$$




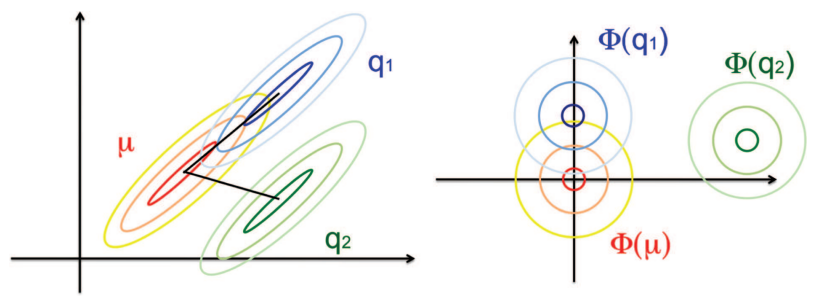

Fig. 6. Selection of discriminative visual elements - interpretation using linear discriminant analysis. Left: The negative data distribution (centered at $\mu$ ) and two example positive data distributions $\left(q_{1}\right.$ and $\left.q_{2}\right)$ are modeled as Gaussians with different means but the same covariance. Right: After "whitening", the negative data is centered at the origin with unit covariance. For fixed negative data, the classifier defined by $q_{2}$ is clearly more discriminative than the classifier defined by $q_{1}$, as measured by the overlap of the positive and negative data distributions. In the whitened space, this overlap can be measured by the Euclidean distance of the (whitened) mean of the positive data points from the origin. Note that in the original nonwhitened space (left) the means of $q_{1}$ and $q_{2}$ are at the same distance from the mean of the negative data $\mu$.

can be obtained in closed form as

$$
w_{L D A}=\Sigma^{-1}\left(q-\mu_{n}\right),
$$

and

$$
b_{L D A}=\frac{1}{2}\left(\mu^{T} \Sigma^{-1} \mu-q^{T} \Sigma^{-1} q\right) .
$$

Note that the matching score (8) can also be expressed using the whitening transformation defined in (7) as

$$
s_{L D A}(x)=\Phi(q)^{T} \Phi(x)-\frac{1}{2}\|\Phi(q)\|^{2},
$$

where the first term is a dot-product between whitened $q$ and $x$, and the second term is an additive normalization factor reducing the matching score for $q$ vectors with large whitened norm. It is interesting to note that under the Gaussian assumptions of LDA, the squared whitened norm $\|\Phi(q)\|^{2}$ can be interpreted as the Bhattacharyya distance [Kailath 1967] measuring the "overlap" between the Gaussian representing the negative data and the Gaussian representing the positive example $q$. Discriminative visual elements $q$ with large $\|\Phi(q)\|$ (as described in Section 4.2.2) correspond to "unusual" examples far from the distribution of the negative data. This intuition is illustrated in Figure 6.

4.2.4 Discussion. Classifiers obtained by minimizing the least squares cost function (2) or satisfying the LDA ratio test can be used for matching a candidate visual element $q$ to a painting as described in Eq. (1). Note that the decision hyperplanes obtained from the least squares regression, $w_{L S}$, and linear discriminant analysis, $w_{L D A}$, are parallel. As a consequence, for a particular visual element $q$ the ranking of matches according to the matching score (1) would be identical for the two methods. In other words, in an object detection setup [Dalal and Triggs 2005; Hariharan et al. 2012; Gharbi et al. 2012] the two methods would produce identical precisionrecall curves. In our matching setup, for a given $q$ the best match in a particular painting would be identical for both methods. The actual value of the score, however, becomes important when comparing matching scores across different visual element detectors $q$. In object detection, the score of the learned classifiers is typically calibrated on a held-out set of labeled validation examples [Malisiewicz et al. 2011].
4.2.5 Calibrated Discriminative Matching. We have found that calibration of matching scores across different visual elements is important for the quality of the final matching results. Next we describe a procedure to calibrate matching scores without the need of any labelled data. First, we found (Section 6.4.3) that the matching score obtained from LDA produces significantly better matching results than matching via least squares regression. Nevertheless, we found that the raw uncalibrated LDA score favors low-contrast image regions, which have an almost zero HOG descriptor. To avoid this problem, we further calibrate the LDA score (8) by subtracting a term that measures the score of the visual element $q$ matched to a low-contrast region, represented by zero (empty) HOG vector

$$
\begin{aligned}
s_{\text {calib }}(x) & =s_{L D A}(x)-s_{L D A}(0) \\
& =(q-\mu)^{T} \Sigma^{-1} x .
\end{aligned}
$$

This calibrated score gives much better results on the dataset of Hauagge and Snavely [2012] as shown in Section 6.4.3 and significantly improves matching results on our dataset of historical photographs and nonphotographic depictions. Finally, since we wish to obtain matches that are both (i) nonambiguous and (ii) have a high matching score, we perform the following two-step procedure to select candidate visual element matches for a given depiction. First, we apply all visual element detectors on the depiction and take the top 200 detections sorted according to the first-to-second nearest-neighbor ratio [Lowe 2004], using the calibrated similarity score (12). This selects the most nonambiguous matches. Second, we sort the 200 matches directly by score (12) and consider the top 25 matches to compute the camera viewpoint as described in Section 5 .

4.2.6 Summary. Candidate visual elements $\left\{q_{i}\right\}$ are obtained by finding local maxima of (6), which is inversely proportional to the least squares regression training error given by (5) as described in Section 4.2.2. Visual elements are then matched to a painting using the two-step matching procedure described in Section 4.2.5 that uses the calibrated LDA score (12).

\subsection{Filtering Elements Unstable Across Viewpoint}

Here we wish to discard elements that cannot be reliably detected in close-by rendered views. This filtering criterion removes many unstable elements that are, for example, ambiguous because of repeated structures in the rendered view or cover large depth discontinuities and hence significantly change with viewpoint.

We define close-by views based on the visual overlap of imaged $3 \mathrm{D}$ structures rather than, for example, the distance between camera centers. In detail, to measure visual overlap between views $V^{1}, V^{2}$ we define the following score

$$
S\left(V^{1}, V^{2}\right)=\frac{1}{|\mathcal{V}|} \sum_{\left\{x_{i}^{1}, x_{i}^{2}\right\} \in \mathcal{V}} e^{-\frac{\left(x_{i}^{1}-x_{i}^{2}\right)^{2}}{2 \sigma_{x}^{2}}-\frac{1}{2 \sigma_{d}^{2}} \frac{\left(d\left(x_{i}^{1}\right)-d\left(x_{i}^{2}\right)\right)^{2}}{\frac{1}{2}\left(d\left(x_{i}^{1}\right)+d\left(x_{i}^{2}\right)\right)^{2}},}
$$

where $\left\{x_{i}^{1}, x_{i}^{2}\right\} \in \mathcal{V}$ is the set of corresponding points (pixels) in view $V^{1}$ and $V^{2}$, respectively, $x_{i}^{j}$ is the location of pixel $i$ in view $j, d\left(x_{i}^{j}\right)$ is the depth (distance to the 3D model) at pixel $i$ in view $j$, and $\sigma_{x}$ and $\sigma_{d}$ are parameters. The first term in the exponent measures the squared image distance between the corresponding pixels. The second term in the exponent measures the difference between the depths at the corresponding pixel locations normalized by their average depth. The per-pixel scores are then averaged over all corresponding pixels in the two views. The score is one if the two 


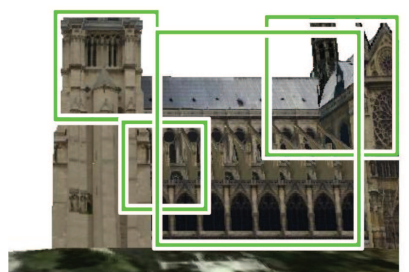

(a) top 4 stable patches

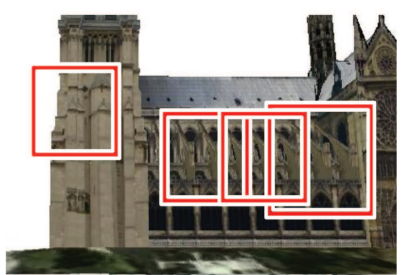

(b) top 4 unstable patches
Fig. 7. Filtering elements unstable across viewpoint. Examples of top stable (left) and unstable (right) visual elements detected in one of the rendered views. Unstable elements are typically detected on repeated structures or occlusion boundaries and are removed.

views are identical and zero if the two views have no visual overlap. In our case, two views are deemed "close-by" if their visual overlap score is greater than 0.4 . Note that the score depends on camera positions as well as the 3D structure as it measures differences between projected $3 \mathrm{D}$ points in the image plane. As a result, the score is, for example, less sensitive to small camera translations if the camera is looking at a far-away scene. We found that good values for the parameters are $\sigma_{d}=0.3$ and $\sigma_{x}=(W+H) / 10$, where $W$ and $H$ are, respectively, the width and height of the rendered views.

Here we evaluate if each candidate discriminative visual element obtained as described in Section 4.2 is stable. Equipped with the previous definition of nearby views, we test if each candidate visual element can be correctly detected in the set of nearby views using the ground-truth locations of the candidate element obtained from the knowledge of the 3D model. In detail, we first select the nearby views in which the visual element is fully visible. Then, we attempt to localize the visual element in each view by applying the corresponding linear detector given by Eq. (12) in a sliding-window fashion.

To suppress potential repeated structures, we require that the ratio between the score of the first and second highest-scoring detection in the image is larger than a threshold of 1.04, similar to, Lowe [2004]. We keep visual elements that are successfully detected in more than $80 \%$ of the nearby views. Examples of stable and unstable visual elements are shown in Figure 7.

This procedure typically results in several thousand selected elements for each architectural site. Examples of the final visual elements obtained by the proposed approach are shown in Figure 8.

\section{RECOVERING VIEWPOINT}

In this section we describe how, given the set of discriminative visual elements gleaned from the 3D model, to recover the viewpoint and intrinsic parameters of an input painting or historical photograph with respect to the $3 \mathrm{D}$ model. We assume that the paintings are perspective scene renderings and seek to recover the following set of parameters via camera resectioning [Hartley and Zisserman 2004]: camera center, camera rotation, focal length, and principal point. Our alignment procedure consists of a coarse alignment step using the recovered discriminative visual elements, followed by an optional fine alignment procedure for the case of perspectively correct scene depictions (e.g., photographs, paintings with little/no perspective drawing errors).

For detection, each discriminative visual element takes as input a 2D patch from the painting and returns as output a 3D location $\mathbf{X}$ on the 3D model, a plane representing the patch extent on the 3D model centered at $\mathbf{X}$, and a detector response score indicating the quality of the appearance match. Following the matching

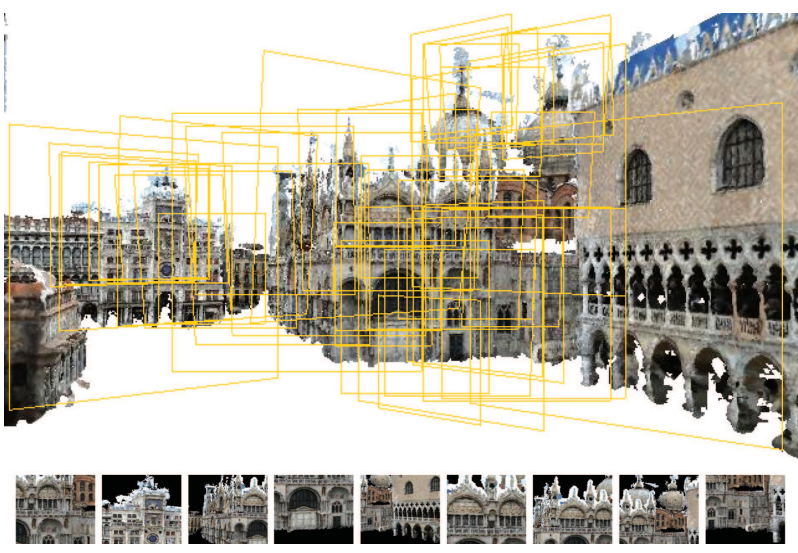

Fig. 8. Examples of selected visual elements for a 3D site. Top: Selection of top-ranked 50 visual elements visible from this specific view of the site. Each element is depicted as a planar patch with an orientation of the plane parallel to the camera plane of its corresponding source view. Bottom: Subset of 9 elements shown from their original viewpoints. Note that the proposed algorithm prefers visually salient scene structures such as the two towers in the top-right or the building in the left part of the view. In contrast, some repetitive and nonsalient scene structures in the right portion of the picture are ignored.

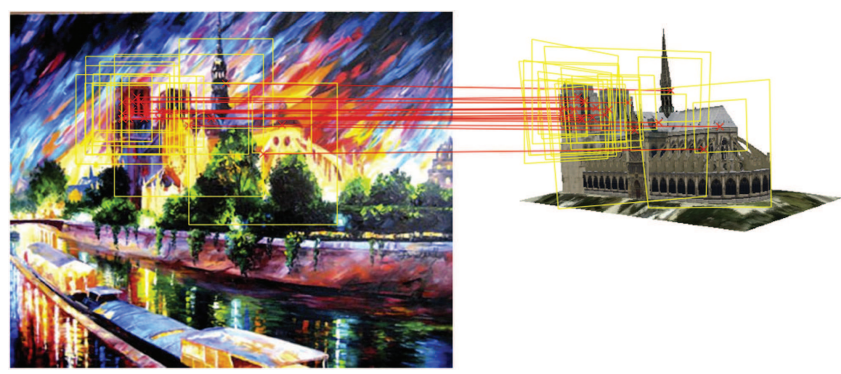

Fig. 9. Illustration of coarse alignment. We use the recovered discriminative visual elements to find correspondences between the input scene depiction and 3D model. Shown is the recovered viewpoint and inlier visual elements found via RANSAC. Notice that the visual elements yield inliers across the entire visible part of the site. Painting courtesy of Daniel Wall.

procedure described in Section 4.2.5, we form a set of 25 putative discriminative visual element matches. From each putative visual element match we obtain 5 putative point correspondences by taking the 2D/3D locations of the patch center and its four corners. The patch corners provide information about the patch scale and the planar location on the 3D model, and has been shown to work well for structure from motion with planar constraints [Szeliski and Torr 1998]. We use RANSAC [Fischler and Bolles 1981] to find the set of inlier correspondences to a restricted camera model where the camera intrinsics are fixed to initial values, with the focal length set to the image diagonal length and the principal point set to the center of the image. We use a RANSAC inlier threshold set to $1.5 \%$ of the image diagonal length to recover the camera center and rotation. The recovered viewpoint forms a coarse alignment of the input depiction to the 3D model, which is shown in Figure 9.

For perspectively correct scene depictions, we can further improve the alignment by recovering all of the camera parameters. This is achieved by densely aligning the input depiction to the 3D model with an ICP-like fine alignment procedure. At each iteration 


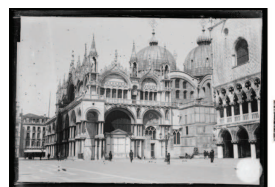

(a) historical photo

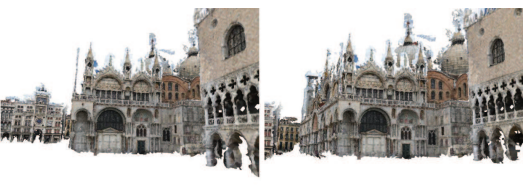

(b) coarse alignment (c) fine alignment
Fig. 10. ICP-like fine alignment for perspectively correct scene depictions. Given the coarse alignment, at each iteration we find a dense set of inlier correspondences with RANSAC for HOG features computed over a grid. We then update the viewpoint and iterate. Notice that the fine alignment step produces a tighter, more accurate fit. Photograph courtesy of la Médiathèque de l'architecture et du patrimoine.

we compute a set of dense correspondences using HOG features computed in a grid over the entire image. Namely, for all HOG descriptors in a given scale in the HOG pyramid, we search for the best match in a local $5 \times 5$ window using L2 distance. We optimize over all camera parameters and find inlier correspondences via RANSAC over the dense set of correspondences. An updated view is rendered from the 3D model and the entire procedure is repeated at a finer scale in the HOG pyramid. In this way, large misalignments are corrected at the beginning of the procedure, with minor adjustments made at the end. In addition to the putative correspondences obtained via dense HOG matching at each iteration, we also include inlier correspondences from previous ICP iterations during RANSAC and fitting, This avoids the camera parameters from overfitting to a particular region in the input depiction. We illustrate the output of the fine alignment procedure in Figure 10.

\section{RESULTS AND VALIDATION}

In this section, we first describe our dataset of nonphotographic depictions and historical photographs in, Section 6.1. We then provide qualitative (6.2) and quantitative (6.3) results of our full pipeline. Finally, we provide a detailed analysis of our algorithm in Section 6.4.

\subsection{Dataset for 2D-to-3D Alignment}

We have collected a set of human-generated 3D models from Trimble 3D Warehouse for the following architectural landmarks: Notre Dame of Paris, Trevi Fountain, and San Marco's Basilica. The Trimble 3D Warehouse models for these sites consist of basic primitive shapes and have a composite texture from a set of images. In addition to the Trimble 3D Warehouse models, we also consider a 3D model of San Marco's Square that was reconstructed from a set of photographs using dense multiview stereo [Furukawa et al. 2010]. Note that while the latter 3D model has more accurate geometry than the Trimble 3D Warehouse models, it is also much noisier along the model boundaries.

We have also collected from the Internet 85 historical photographs and 252 nonphotographic depictions of the sites. We separated the nonphotographic depictions into the following categories: "drawings" (60 images), "engravings" (45 images), and "paintings" (147 images). The drawings category includes color renderings and the paintings category includes different rendering styles, such as watercolors, oil paintings, and pastels. Table I shows the number of images belonging to each category across the different sites.

\subsection{Qualitative Results}

Figures 11 and 12 show example alignments of historical photographs and nonphotographic depictions, respectively. Notice that
Table I. Statistics of Our Collected Dataset of Historical Photographs and Nonphotographic Depictions for the Evaluated Architectural Landmarks

\begin{tabular}{c|c|c|c|c|c}
\hline & $\begin{array}{c}\text { S. Marco } \\
\text { Square }\end{array}$ & $\begin{array}{c}\text { S. Marco } \\
\text { Basilica }\end{array}$ & $\begin{array}{c}\text { Trevi } \\
\text { Fountain }\end{array}$ & $\begin{array}{c}\text { Notre } \\
\text { Dame }\end{array}$ & Total \\
\hline Hist. photos & 44 & $(30)$ & 0 & 41 & $\mathbf{8 5}$ \\
\hline Paintings & 61 & $(41)$ & 34 & 52 & $\mathbf{1 4 7}$ \\
\hline Drawings & 21 & $(19)$ & 5 & 34 & $\mathbf{6 0}$ \\
\hline Engravings & 15 & $(9)$ & 10 & 20 & $\mathbf{4 5}$ \\
\hline Total & $\mathbf{1 4 1}$ & $\mathbf{( 9 9 )}$ & $\mathbf{4 9}$ & $\mathbf{1 4 7}$ & $\mathbf{3 3 7}$ \\
\hline
\end{tabular}

Note that the depictions of San Marco Basilica are also included in the set for San Marco Square, with the total (bottom row) counting the number of unique depictions in our dataset.

the depictions are reasonably well aligned, with regions on the $3 \mathrm{D}$ model rendered onto the corresponding location for a given depiction. We are able to cope with a variety of viewpoints with respect to the 3D model as well as different depiction styles. Our approach succeeds in recovering the approximate viewpoint in spite of these challenging appearance changes and the varying quality of the $3 \mathrm{D}$ models. In Figure 13 we show alignments to a set of challenging examples where the assumption of a perspective rendering is significantly violated, but the proposed approach was still able to recover a reasonable alignment. Notice the severe nonperspective scene distortions, drawing errors, and major architectural differences (e.g., a part of the landmark may take a completely different shape).

Figure 14 shows the camera frusta for the recovered approximate painting viewpoints. Notice that our system is able to recover viewpoints that are to the rear of the main facade of the Notre Dame cathedral, which has not been possible in prior work [Snavely et al. 2006] due to the lack of reconstructed structure in these areas. Recovering approximate camera viewpoints for paintings and historical photographs opens up the possibility of large-scale automatic computational rephotography for such depictions [Bae et al. 2010]. The video http://www.di.ens.fr/willow/research/ painting_to_3d/data/ND.mp4 shows an example of a virtual tour of an architectural site transitioning between viewpoints of different images in 3D in a similar manner to Snavely et al. [2006], but here done for the challenging case of historical photographs, nonphotographic depictions, and only an approximate 3D model from Trimble 3D Warehouse. Many architectural sites now have 3D models geolocated on a map, which, combined with the proposed approach, would enable geolocating historical photographs and nonphotographic depictions [Shrivastava et al. 2011], for example, navigation and exploration of nonphotorealistic depictions (as shown in the video above or, coarsely aligned manually, at http://www. whatwasthere.com) or in situ guided tours of historical imagery using mobile or wearable display devices.

\subsection{Quantitative Evaluation}

In the following we give details of the performed user study, report quantitative results across the $3 \mathrm{D}$ sites and depiction styles, and compare performance with several baseline methods.

To quantitatively evaluate the goodness of our alignments, we have conducted a user study via Amazon Mechanical Turk. The workers were asked to judge the viewpoint similarity of the resulting alignments to their corresponding input depictions by categorizing the viewpoint similarity as either a: (a) good match, (b) coarse match, or (c) no match, illustrated in Figure 15. We asked five different workers to rate the viewpoint similarity for each depiction and we report the majority opinion. 

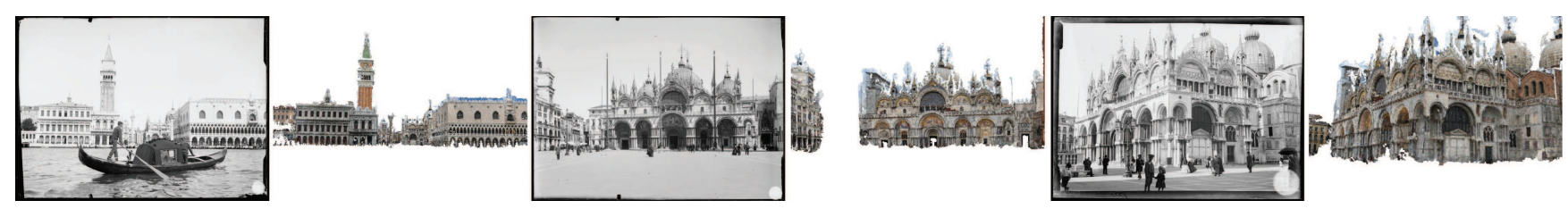

Fig. 11. Alignment of historical photographs of San Marco's Square to their respective 3D models. Photographs courtesy of la Médiathèque de l'architecture et du patrimoine.
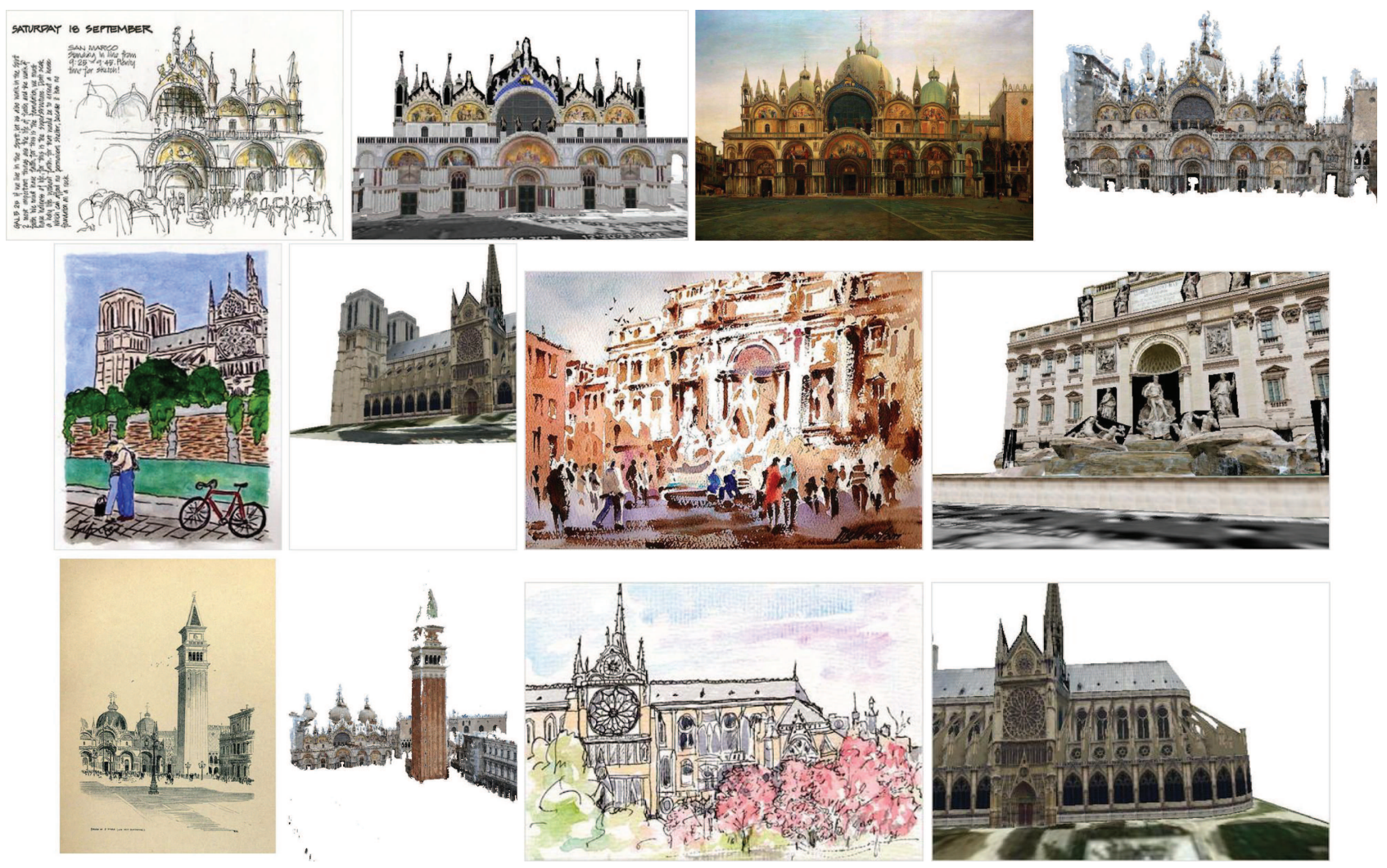

Fig. 12. Example alignments of nonphotographic depictions to 3D models. Notice that we are able to align depictions rendered in different styles and having a variety of viewpoints with respect to the 3D models. Images courtesy of Liz Steel, Fifi Flowers, Woom Lam Ng, and Noreen Wessling. More results are available at the project Web site http://www.di.ens.fr/willow/research/painting_to_3d/.

Table II shows the performance of our algorithm for the different $3 \mathrm{D}$ sites considered in this work. As expected, the performance varies to some extent across the different models depending on their size, quality, and the difficulty of the matching task. However, the failure (no match) rate remains consistently below $30 \%$.

Table III shows the performance of our algorithm for different depiction styles averaged across the $3 \mathrm{D}$ sites. Interestingly, the results are fairly consistent across different depiction styles.

Finally, Table IV compares the performance of our algorithm to several baseline methods for the 141 depictions of San Marco Square-the largest 3D model in our dataset with $45 \mathrm{~K}$ sampled viewpoints. We compare our algorithm against the following four baselines: (i) SIFT on rendered views, (ii) viewpoint retrieval (corresponding to the coarse alignment step of Russell et al. [2011]), (iii) Exemplar SVM [Shrivastava et al. 2011], and (iv) mid-level painting visual elements that, similar to Singh et al. [2012], learns mid-level visual elements directly from paintings, rather than the 3D model. The implementation details of each baseline are given next.

For the SIFT on rendered views baseline we extract and match SIFT descriptors computed at interest points across scale [Lowe 2004] over each input depiction and all rendered views. We use orientation-sensitive descriptors as we found them more reliable than orientation-invariant descriptors in practice. We perform geometric verification by finding inliers to an affine homography between the input depiction and each rendered viewpoint. Then, we take the rendered viewpoint with the most inliers and perform camera resectioning with RANSAC using the SIFT putative matches for that view. We return as output a rendering of the final resectioned viewpoint. Note that the matching procedure is not standard since it is extracting descriptors from rendered views, which accounts for viewpoint changes. In other words, the SIFT matching 

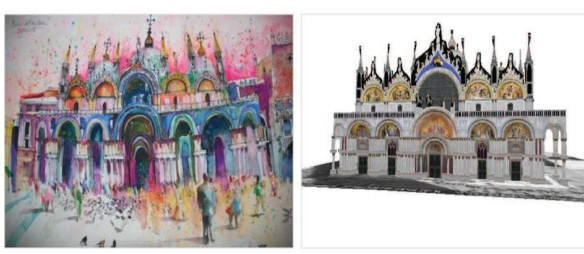

(a) scene distortion

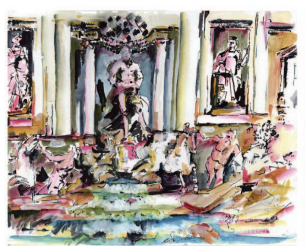

(b) drawing and $3 \mathrm{D}$ errors
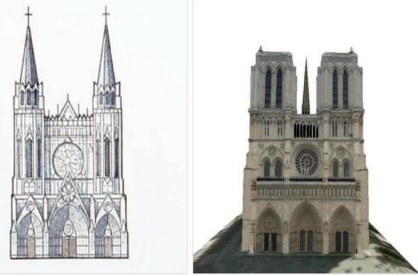

(c) major structural differences

Fig. 13. Challenging examples successfully aligned by our method where the assumption of a perspective scene rendering is violated. Note that the drawing in (c) is a completely different cathedral. Image in (a) courtesy of Blythe Scott and in (b) courtesy of Ginette Callaway.

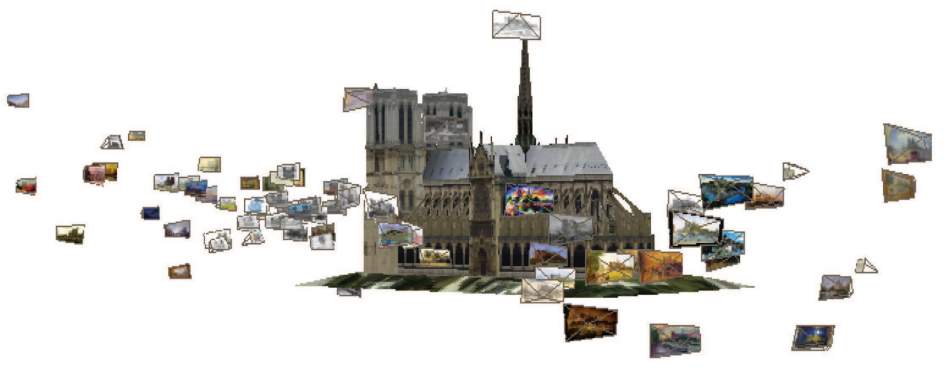

(a) Notre Dame of Paris

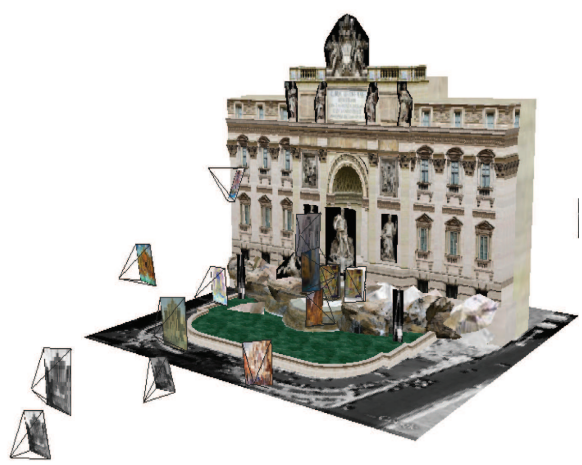

(b) Trevi Fountain

Fig. 14. Trimble 3D Warehouse models and camera frusta depicting the recovered viewpoints of the paintings.

Table II. Viewpoint Similarity User Study of Our Algorithm across Different Sites

\begin{tabular}{c|c|c|c}
\hline & $\begin{array}{c}\text { Good } \\
\text { match }\end{array}$ & $\begin{array}{c}\text { Coarse } \\
\text { match }\end{array}$ & $\begin{array}{c}\text { No } \\
\text { match }\end{array}$ \\
\hline S. Marco Square & $51 \%$ & $21 \%$ & $28 \%$ \\
\hline S. Marco Basilica & $45 \%$ & $39 \%$ & $15 \%$ \\
\hline Trevi Fountain & $55 \%$ & $20 \%$ & $24 \%$ \\
\hline Notre Dame & $65 \%$ & $27 \%$ & $9 \%$ \\
\hline Average & $\mathbf{5 5 \%}$ & $\mathbf{2 7 \%}$ & $\mathbf{1 8 \%}$ \\
\hline
\end{tabular}

step does not need to be viewpoint invariant as we are matching to a similar viewpoint from the rendered set. This baseline is similar in spirit to matching with Viewpoint-Invariant Patches (VIP) [Wu et al. 2008], except no depth or rectification is needed for the input depictions. This baseline performs reasonably well, having $40 \%$ good alignments compared with $51 \%$ for our algorithm. The good performance is largely due to alignments of historical photographs $(70 \%$ versus $50 \%$ for our method). However, if historical photographs are removed from the dataset, the SIFT on rendered views baseline drops to $27 \%$ good alignments, while our algorithm still achieves $52 \%$ good alignments.

The viewpoint retrieval baseline consists of matching a global Gist descriptor [Oliva and Torralba 2001] extracted for each input depiction and all rendered views. The Gist descriptors are compared using L2 distance and the view corresponding to the minimum distance is returned. The Gist descriptor is sensitive to viewpoint, with the matching procedure corresponding to the coarse alignment step of Russell et al. [2011]. Our method clearly outperforms the
Table III. Viewpoint Similarity User Study of Our Algorithm across Different Depiction Styles

\begin{tabular}{c|c|c|c}
\hline & $\begin{array}{c}\text { Good } \\
\text { match }\end{array}$ & $\begin{array}{c}\text { Coarse } \\
\text { match }\end{array}$ & $\begin{array}{c}\text { No } \\
\text { match }\end{array}$ \\
\hline Historical photographs & $59 \%$ & $20 \%$ & $21 \%$ \\
\hline Paintings & $53 \%$ & $30 \%$ & $18 \%$ \\
\hline Drawings & $52 \%$ & $29 \%$ & $19 \%$ \\
\hline Engravings & $57 \%$ & $26 \%$ & $17 \%$ \\
\hline Average & $\mathbf{5 5 \%}$ & $\mathbf{2 7 \%}$ & $\mathbf{1 8 \%}$ \\
\hline
\end{tabular}

Table IV. Viewpoint Similarity User Study - Comparison with Baselines on the San Marco Square 3D Site

\begin{tabular}{c|c|c|c}
\hline & $\begin{array}{c}\text { Good } \\
\text { match }\end{array}$ & $\begin{array}{c}\text { Coarse } \\
\text { match }\end{array}$ & $\begin{array}{c}\text { No } \\
\text { match }\end{array}$ \\
\hline SIFT on rendered views & $40 \%$ & $26 \%$ & $33 \%$ \\
\hline Viewpoint retrieval [Russell et al. 2011] & $1 \%$ & $39 \%$ & $60 \%$ \\
\hline Exemplar SVM [Shrivastava et al. 2011] & $34 \%$ & $18 \%$ & $48 \%$ \\
\hline Mid-level painting visual elements & $33 \%$ & $29 \%$ & $38 \%$ \\
\hline 3D discrim. visual elements (ours) & $\mathbf{5 1 \%}$ & $\mathbf{2 1 \%}$ & $\mathbf{2 8 \%}$ \\
\hline
\end{tabular}

viewpoint retrieval baseline, mainly because the sampled rendered views fail to cover the enormous space of all possible viewpoints. Matching the global image-level Gist descriptor would require much denser and wider sampling of views.

To reduce the viewpoint coverage issue, we explore as a baseline the Exemplar-SVM approach of Shrivastava et al. [2011]. For this 


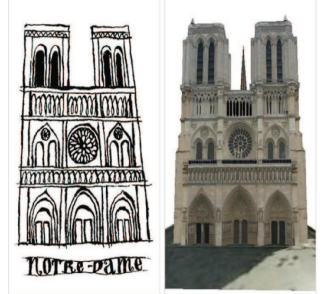

(a) good match

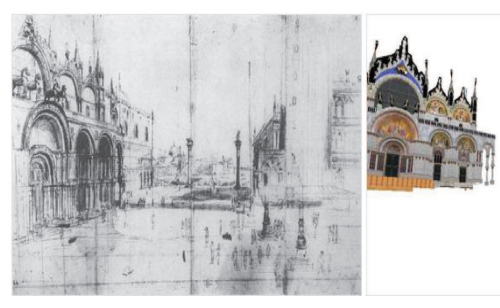

(b) coarse match

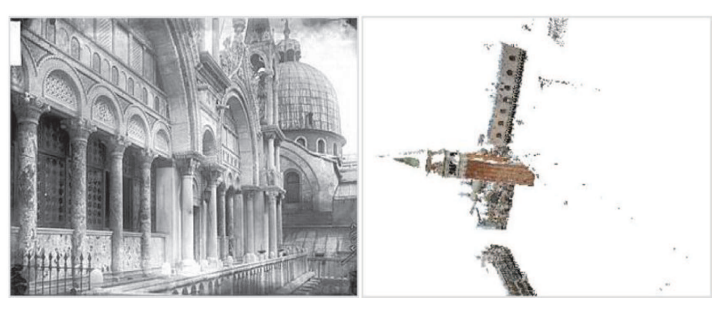

(c) no match

Fig. 15. Alignment evaluation criterion. We asked workers on Amazon Mechanical Turk to judge the viewpoint similarity of the resulting alignment to the input depiction. The workers were asked to categorize the viewpoint similarity into one of three categories: (a) good match-the two images show a roughly similar view of the building; (b) coarse match—-the view may not be similar, but the building is roughly at the same location in both images, not upside down, and corresponding building parts can be clearly identified; (c) no match-the views are completely different, for example, upside down, little or no visual overlap. Image in (a) courtesy of Maral Sassouni and in (c) courtesy of la Médiathèque de l'architecture et du patrimoine.

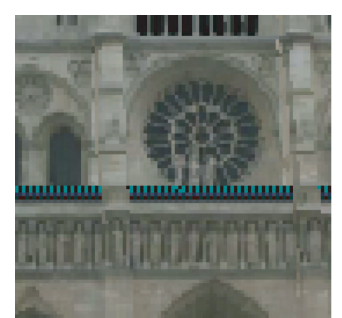

(a) original rendering

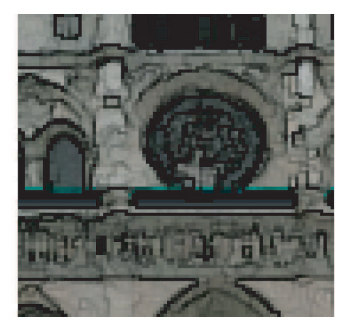

(b) drawing style

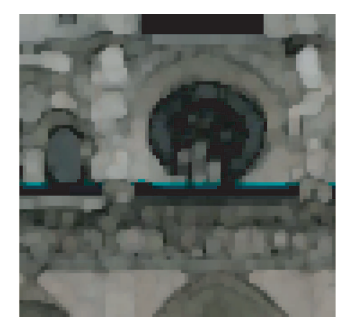

(c) painting style

Fig. 16. Viewpoint rendering styles. We explored the possibility of rendering viewpoints from the 3D model in different styles by applying style filters within Photoshop CS4 to the rendered views.

a single Exemplar SVM detector is trained for each input depiction and is subsequently matched across all scales and 2D locations in sliding-window fashion in the rendered views. While the performance improves over Gist matching, nonetheless the results remain limited since the approach cannot handle partial occlusions and significant deformations that are common in nonphotorealistic depictions. Moreover, the procedure is computationally expensive since an SVM detector is trained with hard negative mining for each input painting, with the resulting detector run in a sliding-window fashion over all rendered views. In contrast, our approach learns offline a few thousand visual element detectors that compactly summarize an entire architectural site. At runtime, only the learned visual elements are applied to the input depiction.

To overcome the issues with partial occlusion and significant deformations, but keeping the idea of matching the input painting to the rendered views, we extract mid-level visual elements directly from the input paintings without any explicit knowledge of the 3D model. In detail, we extract 25 mid-level discriminative visual elements from each input painting using the approach presented in Section 4.2.2. The painting visual elements are then matched in a sliding-window fashion to all rendered views. For each rendered view, inlier point correspondences are recovered via camera resectioning with RANSAC over the maximal detector responses. The resectioned view that yields the largest number of inliers is rendered and returned. Note that this baseline is similar in spirit to learning mid-level patches [Doersch et al. 2012; Singh et al. 2012] from the input paintings without explicit knowledge of the 3D model. While this baseline further improves over Exemplar SVM (38\% versus $48 \%$ failures), it does not outperform our method mainly because it cannot combine visual element detections from multiple views
Table V. Evaluation of Different 3D Model

\begin{tabular}{c|c|c|c}
\multicolumn{4}{c}{ Rendering Styles } \\
\hline & $\begin{array}{c}\text { Good } \\
\text { match }\end{array}$ & $\begin{array}{c}\text { Coarse } \\
\text { match }\end{array}$ & $\begin{array}{c}\text { No } \\
\text { match }\end{array}$ \\
\hline Drawing style & $61 \%$ & $12 \%$ & $27 \%$ \\
\hline Painting style & $54 \%$ & $18 \%$ & $28 \%$ \\
\hline Original rendering & $\mathbf{6 5 \%}$ & $\mathbf{2 7 \%}$ & $\mathbf{9 \%}$ \\
\hline
\end{tabular}

available to our method via the $3 \mathrm{D}$ model. Similar to Exemplar SVM, an additional drawback of this baseline is the high computational cost as visual elements from each painting must be run densely across all rendered views.

\subsection{Algorithm Analysis}

In this section we evaluate variants of the three steps of our algorithm: viewpoint rendering style, visual element selection, and visual element matching. Finally, we show and analyze the main failure modes.

6.4.1 Viewpoint Rendering Style. Since our goal is to align a 3D model to nonphotorealistic depictions, we explored the possibility of applying different rendering styles during the viewpoint rendering step of our algorithm. We applied the "watercolor" and "accentuated edges" style filters from Photoshop CS4 to our rendered views to generate, respectively, a "painting-like" and a "drawing-like" style. Example filter outputs are shown in Figure 16. We quantitatively evaluate the output of our full system (using the style filters during rendering) on 147 depictions of the Notre Dame site via a user study 

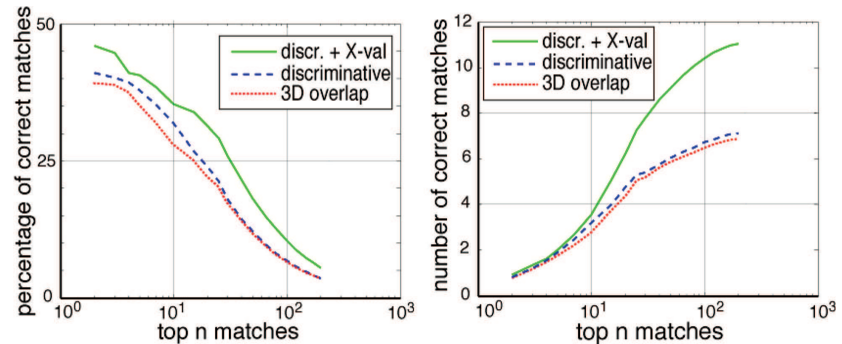

Fig. 17. Evaluation of visual element selection. The average percentage (left) and number (right) of correct matches as a function of the top $n$ matches. See text for details.

on Amazon Mechanical Turk. Results are summarized in Table V. Both styles result in a decrease of the overall matching performance compared to the original rendering. However, when results are split by depiction (not reported in Table V) the drawing style results in a small increase of matching performance on drawings (68\% good matches versus $62 \%$ good matches with the original rendering). While this difference amounts to only 3 additional matched depictions, it opens up the possibility of learning a vocabulary of visual elements specific for each rendering style.

6.4.2 Visual Element Selection. Here we evaluate benefits of the proposed discriminative visual element selection. To measure the improvement in the quality of the selected visual elements we compute the percentage of correct matches (inliers). We consider only the San Marco Square 3D model and the ground truth is obtained by visual inspection of the resulting alignments; only correct matches from the good and coarse alignments are considered as ground-truth inliers. The percentage of inliers gives a finer indication of the quality of visual elements than the overall percentage of correct alignments measured in the previous section, as RANSAC will often find the correct alignment even from very few correct candidate correspondences. Results are summarized in Figure 17. Here $10 \mathrm{~K}$ discriminative visual elements were learned from $45 \mathrm{~K}$ sampled views. We compare three methods for selecting visual elements from the set of rendered views: The "3D overlap" (red) method selects visual elements that significantly overlap the 3D model in rendered views, that is, where at least $50 \%$ of the HOG support is occupied by the 3D model. $10 \mathrm{~K}$ visual elements are then chosen randomly out of all visual elements that satisfy the 3D model overlap criterion. The "discriminative" (blue) method uses the discriminative selection (Section 4.2.2), but no cross-validation. The "discr. + X-val" (green) uses the proposed discriminative visual element selection (Section 4.2.2) with cross-validation (Section 4.2.5). For example, inspecting Figure 17(a) reveals that within the top 10 matches there are $27.9 \%$ correct matches for the 3D overlap method, $31.9 \%$ for the discriminative selection, and $35.4 \%$ for the discriminative selection with cross-validation. This demonstrates that visual elements selected by the proposed method are more likely to be correctly recovered in the painting.

6.4.3 Visual Element Matching. We evaluate the proposed matching procedure on the "desceval" task from the benchmark dataset collected in Hauagge and Snavely [2012]. The benchmark consists of challenging imagery, such as historical photographs and nonphotographic depictions of architectural landmarks. Pairs of images in the dataset depicting a similar viewpoint of the same landmark have been registered by fitting a homography to manual point correspondences. The task is to find corresponding patches in each image pair. Since the ground-truth correspondence between points
Table VI. Evaluation of Visual Element Matching

\begin{tabular}{c|c}
\hline Matching method & mAP ("desceval”) \\
\hline Local symmetry [Hauagge and Snavely 2012] & 0.58 \\
\hline Least squares regression (Sec. 4.2.2) & 0.52 \\
\hline LDA (Sec. 4.2.3) & 0.60 \\
\hline Ours (Sec. 4.2.5) & $\mathbf{0 . 7 7}$ \\
\hline
\end{tabular}

We report the mean average precision on the "desceval" task from the benchmark dataset of Hauagge and Snavely [2012].
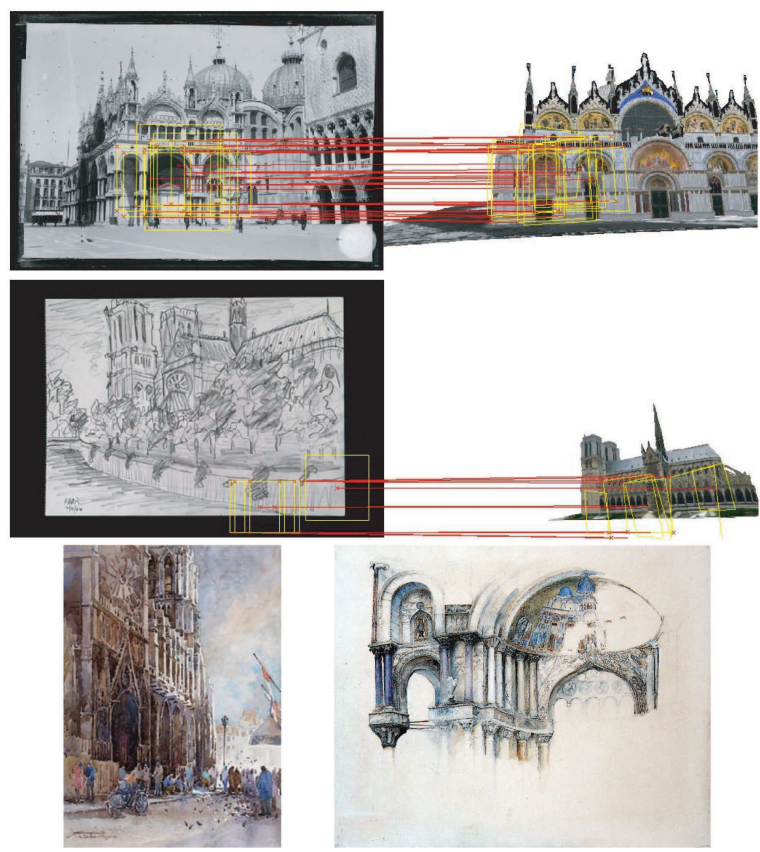

Fig. 18. Example failure cases. Top: large-scale symmetry. Here arches are incorrectly matched on a building with similar front and side facades. Middle: locally confusing image structures. Here the vertical support structures on the cathedral (right) are locally similar by their HOG descriptor to the vertical pencil strokes on the drawing (left). Bottom: Two examples of paintings with unusual viewpoints. Image in the top row courtesy of la Médiathèque de l'architecture et du patrimoine, in the middle row courtesy of Norman Zif, and in the bottom row left courtesy of Woom Lam Ng.

is assumed known via the homography, a precision-recall curve can be computed for each image pair. We report the mean Average Precision (mAP) measured over all image pairs in the dataset.

Following Hauagge and Snavely [2012] we perform matching over a grid of points in the two views, with the grid having 25 pixel spacing. In Table VI we report the mAP for different visual element matching methods for our system, along with the local symmetry feature baseline of Hauagge and Snavely [2012]. Our full system using the calibrated matching score (Section 4.2.5) achieves a mAP of 0.77 , which significantly outperforms both the alternative visual element matching scores obtained by least squares regression (Section 4.2.2) and Linear Discriminant Analysis (LDA, Section 4.2.3), as well as the local symmetry feature baseline.

6.4.4 Failure Modes. We have identified three main failure modes of our algorithm, examples of which are shown in Figure 18. The first is due to large-scale symmetries, for example, when the front and side facade of a building are very similar. This problem 
is difficult to resolve with only local reasoning. For example, the proposed cross-validation step removes repetitive structures visible in the same view but not at different locations of the site. The second failure mode is due to locally confusing image structures, for example, the vertical support structures on the cathedral in Figure 18 (middle) are locally similar (by their HOG descriptor) to the vertical pencil strokes on the drawing. The learned mid-level visual elements have a larger support than typical local-invariant features (such as SIFT) and hence are typically more distinctive. Nevertheless, such mismatches can occur, and in some cases are geometrically consistent with a certain view of the 3D model. The third failure mode is when the viewpoint depicted in the painting is not covered in the set of sampled views. This can happen for unusual viewpoints including extreme angles, large close-ups, or cropped views.

6.4.5 Computational Cost. The most computationally demanding part of our algorithm is the selection of discriminative visual elements, which can be done offline. Our basic rendering engine outputs between 10 to 80 views per minute depending on the 3D model, but modern GPU implementations are capable of much faster rendering speeds. Additionally, it is possible to render the views on-demand only, without ever storing them, which could significantly reduce the storage requirements, specially for large sites. In our Matlab implementation, the visual element selection learning time is dominated by cross-validation. Overall, the algorithm is able to learn about 2,000 elements per hour using 20 cores on a cluster. Note that after the offline learning only the learned visual elements need to be stored. Each element is represented by an 800-dimensional weight vector, together with the 3D location, scale, and orientation of the corresponding planar patch. During the online detection stage, matching 10,000 visual elements to a $450 \times 360$ image takes about 22 minutes. The final camera resectioning takes about 25 seconds. Both timings are on a single 4-cores machine with our Matlab implementation.

\section{CONCLUSION}

We have demonstrated that automatic image-to-3D model alignment is possible for a range of nonphotographic depictions and historical photographs, which represent extremely challenging cases for current local feature matching methods. To achieve this we have developed an approach to compactly represent a 3D model of an architectural site by a set of visually distinct mid-level scene elements extracted from rendered views, and have shown that they can be reliably matched to a variety of photographic and nonphotographic depictions. We have also shown an application of the proposed approach to computational rephotography to automatically find an approximate viewpoint of historical photographs and paintings. This work is just a step towards computational reasoning about the content of nonphotographic depictions. The developed approach for extracting visual elements opens up the possibility of efficient indexing for visual search of paintings and historical photographs (e.g., via hashing of the HOG features as in Dean et al. [2013]), or automatic fitting of complex nonperspective models used in historical imagery [Rapp 2008].

\section{ACKNOWLEDGMENTS}

We are grateful to the anonymous reviewers for their constructive comments, to Alyosha Efros, Guillaume Obozinski, and Jean Ponce for their useful feedback, to Yasutaka Furukawa for providing access to the San Marco 3D model, and to Daniel Hauagge and Noah Snavely for providing the evaluation code for their paper [Hauagge and Snavely 2012]. We also thank all the artists who allowed us to use their work. We credit Podi Lawrence (UK) for the painting in Figure 1(c) and Figure 2, Daniel Wall for the painting in Figure 9, la Médiathèque de l'architecture et du patrimoine for the photographs of Figures 10, 11, 15, and 18, Liz Steel (lizsteel.com), Fifi Flowers and Noreen Wessling for paintings in Figure 12, Woon $\mathrm{Lam} \mathrm{Ng}$ for paintings in Figures 12 and 18, Blythe Scott for the painting in Figure 13(a), Ginette Callaway for the painitng in Figure 13(b), Getty Images for the drawing in Figure 13(c), Maral Sassouni for the drawing in Figure 15(a) and Norman Ziff for a painting in Figure 18. We credit Ahmet Kalafat and Antonino Grasso Cristaudo for the Trimble 3D Warehouse models and Yasutaka Furukawa for the multiview stereo San Marco's Square model.

\section{REFERENCES}

D. Aliaga, P. Rosen, and D. Bekins. 2007. Style grammars for interactive visualization of architecture. IEEE Trans. Vis. Comput. Graph. 13, 4.

G. Baatz, O. Saurer, K. Koser, and M. Pollefeys. 2012. Large scale visual geo-localization of images in mountainous terrain. In Proceedings of the European Conference on Computer Vision.

L. Baboud, M. Cadik, E. Eisemann, and H.-P. Seidel. 2011. Automatic photo-to-terrain alignment for the annotation of mountain pictures. In Proceedings of the Conference on Computer Vision and Pattern Recognition.

F. Bach. and Z. Harchaoui. 2008. Diffrac: A discriminative and flexible framework for clustering. In Advances in Neural Information Processing Systems.

S. Bae, A. Agarwala, and F. Durand. 2010. Computational rephotography. ACM Trans. Graph. 29, 3.

L. Ballan, G. Brostow, J. Puwein, and M. Pollefeys. 2010. Unstructured video-based rendering: Interactive exploration of casually captured videos. ACM Trans. Graph. 29, 4.

C. M. Bishop. 2006. Pattern Recognition and Machine Learning. Springer.

F. Bosche. 2010. Automated recognition of 3D CAD model objects in laser scans and calculation of as-built dimensions for dimensional compliance control in construction. Adv. Engin. Inf. 24, 1, 107-118.

O. Chum and J. Matas. 2006. Geometric hashing with local affine frames. In Proceedings of the Conference on Computer Vision and Pattern Recognition.

N. Dalal and B. Triggs. 2005. Histograms of oriented gradients for human detection. In Proceedings of the Conference on Computer Vision and Pattern Recognition.

T. Dean, M. Ruzon, M. Segal, J. Shlens, S. Vijayanarasimhan, and J. Yagnik. 2013. Fast, accurate detection of 100,000 object classes on a single machine. In Proceedings of the Conference on Computer Vision and Pattern Recognition.

P. E. Debevec, C. J. Taylor, and J. Malik. 1996. Modeling and rendering architecture from photographs. In Proceedings of the $23^{\text {rd }}$ Annual Conference on Computer Graphics and Interactive Techniques (SIGGRAPH' 96). 1-20.

C. Doersch, S. Singh, A. Gupta, J. Sivic, and A. A. Efros. 2012. What makes Paris look like Paris? ACM Trans. Graph. 31, 4.

R. Fan, K. Chang, C. Hsieh, X. Wang, and C. Lin. 2008. Liblinear: A library for large linear classification. J. Mach. Learn. Res. 9, 1, 18711874.

P. F. Felzenszwalb, R. B. Girshick, D. Mcallester, and D. Ramanan. 2010. Object detection with discriminatively trained part based models. IEEE Trans. Pattern Anal. Mach. Intell. 32, 9, 1627-1645.

M. A. Fischler and R. C. Bolles. 1981. Random sample consensus: A paradigm for model fitting with applications to image analysis and automated cartography. Comm. ACM 24, 6, 381-395. 
A. Frome, Y. Singer, F. Sha, and J. Malik. 2007. Learning globally-consistent local distance functions for shape-based image retrieval and classification. In Proceedings of the International Conference on Computer Vision.

Y. Furukawa, B. Curless, S. M. Seitz, and R. Szeliski. 2010. Towards Internet-scale multi-view stereo. In Proceedings of the Conference on Computer Vision and Pattern Recognition.

Y. Furukawa and J. Ponce. 2010. Accurate, dense, and robust multiview stereopsis. IEEE Trans. Pattern Anal. Mach. Intell. 32, 8.

M. Gharbi, T. Malisiewicz, S. Paris, and F. Durand. 2012. A Gaussian approximation of feature space for fast image similarity. Tech. rep. MIT-CSAIL-TR-2012-032. http://people.csail.mit.edu/tomasz/ papers/gharbi_techreport_2012.pdf.

B. Hariharan, J. Malik, and D. Ramanan. 2012. Discriminative decorrelation for clustering and classification. In Proceedings of the European Conference on Computer Vision.

R. I. Hartley and A. Zisserman. 2004. Multiple View Geometry in Computer Vision $2^{\text {nd }}$ Ed. Cambridge University Press.

D. Hauagge and N. Snavely. 2012. Image matching using local symmetry features. In Proceedings of the Conference on Computer Vision and Pattern Recognition.

D. P. Huttenlocher and S. Ullman. 1987. Object recognition using alignment. In Proceedings of the International Conference on Computer Vision.

A. Irschara, C. Zach, J.-M. Frahm, and H. Bischof. 2009. From structurefrom-motion point clouds to fast location recognition. In Proceedings of the Conference on Computer Vision and Pattern Recognition.

A. Jain, A. Gupta, M. Rodriguez, and L. S. Davis. 2013. Representing videos using mid-level discriminative patches. In Proceedings of the Conference on Computer Vision and Pattern Recognition.

M. Juneja, A. Vedaldi, C. V. Jawahar, and A. Zisserman. 2013. Blocks that shout: Distinctive parts for scene classification. In Proceedings of the Conference on Computer Vision and Pattern Recognition.

T. Kailath. 1967. The divergence and Bhattacharyya distance measures in signal selection. IEEE Trans. Comm. Technol. 15, 1, 52-60.

J. Kopf, B. Neubert, B. Chen, M. Cohen, D. Cohen-Or, O. Deussen, M. Uyttendaele, and D. Lischinski. 2008. Deep photo: Model-based photograph enhancement and viewing. ACM Trans. Graph. 27, 5.

G. Levin and P. Debevec. 1999. Rouen revisited - Interactive installation. http://acg.media.mit.edu/people/golan/rouen/.

Y. Li, N. Snavely, D. Huttenlocher, and P. Fua. 2012. Worldwide pose estimation using 3D point clouds. In Proceedings of the European Conference on Computer Vision.

D. Lowe. 1987. The viewpoint consistency constraint. Int. J. Comput. Vis. $1,1,57-72$.

D. G. Lowe. 2004. Distinctive image features from scale-invariant keypoints. Int. J. Comput. Vis. 60, 2, 91-110.
T. Malisiewicz, A. Gupta, and A. A. Efros. 2011. Ensemble of exemplarSVMs for object detection and beyond. In Proceedings of the International Conference on Computer Vision.

P. Musialski, P. Wonka, D. Aliaga, M. Wimmer, L. Van Gool, W. Purgathofer, N. Mitra, M. Pauly, M. Wand, and D. Ceylan, et al. 2012. A survey of urban reconstruction. In Eurographics State of the Art Reports.

A. Oliva and A. Torralba. 2001. Modeling the shape of the scene: A holistic representation of the spatial envelope. Int. J. Comput. Vis. 42, 3, 145-175.

J. Rapp. 2008. A geometrical analysis of multiple viewpoint perspective in the work of Giovanni Battista Piranesi: An application of geometric restitution of perspective. J. Archit. 13, 6 .

B. C. Russell, J. Sivic, J. Ponce, and H. Dessales. 2011. Automatic alignment of paintings and photographs depicting a 3D scene. In Proceedings of the IEEE Workshop on 3D Representation for Recognition (3dRR' 11).

T. Sattler, B. Leibe, and L. Kobbelt. 2011. Fast image-based localization using direct 2D-to-3D matching. In Proceedings of the International Conference on Computer Vision.

G. Schindler, M. Brown, and R. Szeliski. 2007. City-scale location recognition. In Proceedings of the Conference on Computer Vision and Pattern Recognition.

S. Shalev-Shwartz, Y. Singer, N. Srebro, and A. Cotter. 2011. Pegasos: Primal estimated sub-gradient solver for SVM. Math. Program. Series B 127, 1, 3-30.

E. Shechtman and M. Irani. 2007. Matching local self-similarities across images and videos. In Proceedings of the Conference on Computer Vision and Pattern Recognition.

A. Shrivastava, T. Malisiewicz, A. Gupta, and A. A. Efros. 2011. Datadriven visual similarity for cross-domain image matching. ACM Trans. Graph. 30, 6 .

S. Singh, A. Gupta, and A. A. Efros. 2012. Unsupervised discovery of mid-level discriminative patches. In Proceedings of the European Conference on Computer Vision.

J. Sivic and A. Zisserman. 2003. Video Google: A text retrieval approach to object matching in videos. In Proceedings of the International Conference on Computer Vision.

N. Snavely, S. M. Seitz, and R. Szeliski. 2006. Photo tourism: Exploring photo collections in 3d. ACM Trans. Graph. 25, 3, 835-846.

R. Szeliski. 2006. Image alignment and stitching: A tutorial. Foundat. Trends Comput. Graph. Vis. 2, 1, 1-104.

R. Szeliski and P. Torr. 1998. Geometrically constrained structure from motion: Points on planes. In European Workshop on 3D Structure from Multiple Images of Large-Scale Environments (SMILE'98).

C. Wu, B. Clipp, X. Li, J.-M. Frahm, and M. Pollefeys. 2008. 3D model matching with viewpoint invariant patches (VIPs). In Proceedings of the Conference on Computer Vision and Pattern Recognition.

Received September 2013; accepted November 2013 